be increasing and there is little information about the clinical and epidemiological characteristics of this group of patients.

Objectives: To compare basic demographics of a cohort of deceased sarcoid patients with a non-deceased sarcoid population, and to describe the presenting organ involvement among the deceased sarcoid population.

Methods: Patients seen at the Stanford University Hospital and Clinics from 2009-2017 who were $>18$ years of age with at least one ICD-9 or 10 diagnosis of sarcoidosis and at least one clinical note with the term "sarcoidosis" were identified by EMR. After determining the demographics of this cohort, deceased patients were identified and an extensive chart review of these subset of patients was conducted. The project was approved by Stanford's Institutional Review Board.

Results: 1190 adult patients with an ICD code for sarcoidosis were identified Demographic comparison between the non-deceased and deceased population is summarised in the table 1 below.

\begin{tabular}{lcc}
\hline & Non-deceased sarcoid patients & Deceased sarcoid patients \\
\hline Total patients & 1086 & 104 \\
Gender: & $660(60.8 \%)$ & $59(56.7 \%)$ \\
Females & $426(39.2 \%)$ & $45(43.3 \%)$ \\
Males & & \\
Race: & $67(6.2 \%)$ & $1(0.9 \%)$ \\
Asian & $197(18.1 \%)$ & $26(25 \%)$ \\
Black & $596(54.9 \%)$ & $45(43.2 \%)$ \\
White & $6(0.6 \%)$ & $0(0 \%)$ \\
Native American & $128(11.8 \%)$ & $18(17.4 \%)$ \\
Others & $91(8.4 \%)$ & $14(13.5 \%)$ \\
Unknown & & \\
Ethnicity & $918(84.5 \%)$ & $80(76.9 \%)$ \\
Non-Hispanic & $67(6.2 \%)$ & $11(0.5 \%)$ \\
Hispanic/Latino & $101(9.3 \%)$ & $13(12.6 \%)$ \\
Unknown & &
\end{tabular}

In comparison with non-deceased patients with sarcoidosis, the proportion of African American population was higher in the deceased population, whereas the proportion of Asians and whites was lower in the deceased population. The percentage of male patients in the deceased subset was slightly higher than the nondeceased population. 65 (62.5\%) of the 104 deceased patients were symptomatic at diagnosis, 6 (5.8\%) were asymptomatic and information was not available for $33(31.7 \%)$ patients. Of the 65 symptomatic patients, presenting organ involvement was either pulmonary $(34,52.3 \%)$, cardiac $(11,16.9 \%)$, cutaneous $(8,12.3 \%)$, neurological(4,6.2\%), ocular(3, 4.6\%), constitutional(5,7.7\%), unknown(1,1.5\%), musculoskeletal(1,1.5\%), renal-calcium related(1,1.5\%), hepatic(2,3\%), laryngeal $(1,1.5 \%)$, nasal and sinus(2,3\%), parotid gland(1,1.5\%), gastrointestinal $(1,1.5 \%)$, or others $(5,7.6 \%)$.

Conclusions: This study compares the demographics of subset of deceased sarcoid patients with a population of non-deceased sarcoid patients, and describes the presenting organ involvement among the deceased sarcoid population. African American males had a higher mortality rate compared to Asians and Caucasians. Pulmonary and cardiac disease represented $69.2 \%$ of organ involvement in the deceased. Our study showed a mortality rate of $8.7 \%$ in the sarcoid population which is in keeping with the newer studies that mortality in patients with sarcoid might be on the rise. With further study of this population, we aim to compare the characteristics of non-deceased sarcoid patients with the deceased patients, including presenting organ involvement, diagnostic workup and treatment regimen and hope to gain a better understanding of the possible reasons for increasing mortality in patients with sarcoidosis.

Disclosure of Interest: None declared

DOI: 10.1136/annrheumdis-2018-eular.6164

\section{THU0628 DIFFERENCES IN CLINICAL MANIFESTATIONS OF BEHCET'S SYNDROME BY GENDER: CROSS- SECTIONAL ANALYSIS IN A UK COHORT}

L. Chadwick ${ }^{1}$, T. Blake ${ }^{2}$, N.J. Goodson ${ }^{1}$, D. Situnayake ${ }^{2}$, R.J. Moots ${ }^{1}$. ${ }^{1}$ Behcet's Syndrome Centre of Excellence, Aintree University Hospital NHS Foundation Trust, Liverpool; ${ }^{2}$ Behcet's Syndrome Centre of Excellence, Sandwell and West Birmingham NHS Foundation Trust, Birmingham, UK

Background: Geographical variations in genotype and phenotype of Behçet's syndrome (BS) are reported. ${ }^{1,2}$ Previous meta-analysis of international cohorts has highlighted differences in clinical manifestations of BS by gender. ${ }^{3} \mathrm{~A}$ comparison of BS clinical manifestations by gender has not been analysed previously in a large UK BS cohort.

Objectives: 1) To compare the clinical manifestations of BS in a UK cohort by gender and 2) to compare this to published international data.
Methods: A retrospective cross sectional analysis was performed using clinical databases at the Liverpool and Birmingham BS Centres of Excellence. Patients with a multi-disciplinary diagnosis of BS or International Study Group (ISG) diagnostic criteria were included. Clinical manifestations and HLA-B51 positivity were compared by gender. T-tests and chi-squared tests were used for continuous and categorical data respectively and a p value of 0.05 or less was considered statistically significant.

Results: 433 patients met inclusion criteria (140 males, 32.3\%). Male patients were younger and had significantly higher rates of ocular and vascular involvement, papulopustular skin rash and HLA-B51 positivity. Female patients had significantly higher rates of genital aphthosis and arthralgia.

\begin{tabular}{|c|c|c|c|}
\hline & Male $(n=140)$ & Female $(n=293)$ & $p$ value \\
\hline Age (years), mean (s.d.) ${ }^{*}$ & $41.2(12.5)$ & $44.1(11.9)$ & 0.02 \\
\hline Recurrent oral aphthosis, $n$ (\%) & $139(99.3)$ & $291(99.3)$ & 0.97 \\
\hline Genital aphthosis, $\mathrm{n}(\%)^{*}$ & $111(79.3)$ & $278(94.9)$ & $<0.01$ \\
\hline Papulopustular skin rash, $\mathrm{n}(\%)^{*}$ & $69(49.3)$ & $107(36.5)$ & 0.01 \\
\hline Erythema nodosum, $\mathrm{n}(\%)$ & $29(20.7)$ & $56(19.1)$ & 0.70 \\
\hline Skin aphthosis $(n, \%)$ & $7(5.0)$ & $8(2.7)$ & 0.23 \\
\hline Uveitis, $\mathrm{n}(\%)^{*}$ & $81(57.9)$ & $99(33.8)$ & $<0.01$ \\
\hline Retinal vasculitis, $\mathrm{n}(\%)^{*}$ & $39(27.9)$ & $36(12.3)$ & $<0.01$ \\
\hline Central nervous system involvement, $\mathrm{n}(\%)$ & $16(11.4)$ & $20(6.8)$ & 0.11 \\
\hline Large vein thrombosis, $\mathrm{n}(\%)^{*}$ & $22(15.7)$ & $19(6.5)$ & $<0.01$ \\
\hline Arterial thrombosis or aneurysm, $\mathrm{n}(\%)$ & $4(2.9)$ & $7(2.4)$ & 0.77 \\
\hline Thrombophlebitis, $\mathrm{n}(\%)^{*}$ & $10(7.1)$ & $5(1.7)$ & $<0.01$ \\
\hline Gastro-intestinal involvement, $\mathrm{n}(\%)$ & $8(5.7)$ & $25(8.5)$ & 0.30 \\
\hline Arthralgia, $\mathrm{n}(\%)^{*}$ & $68(48.6)$ & $180(61.4)$ & 0.01 \\
\hline Ethnicity (British or White), $\mathrm{n}(\%)^{*}$ & $\begin{array}{c}90(64.3) \\
(n=126)\end{array}$ & $\begin{array}{c}238(85.9) \\
(n=277)\end{array}$ & $<0.01$ \\
\hline HLA-B51 positive, $n(\%)^{*}$ & $\begin{array}{c}14(58.3) \\
(n=27)\end{array}$ & $\begin{array}{c}9(20.0) \\
(n=45)\end{array}$ & $<0.01$ \\
\hline
\end{tabular}

*statistically significant

Conclusions: Similar to published international cohorts, male patients with BS in the UK have higher rates of ocular and venous involvement, papulopustular skin rash and HLA-B51 positivity and lower rates of genital aphthosis and arthralgia in comparison to female patients. However, the extent to which these differences are attributable to ethnicity is unclear.

\section{REFERENCES :}

[1] Leonardo NM, McNeil J. Behcet's Disease: Is There Geographical Variation? A Review Far from the Silk Road. Int J Rheumatol 2015: 1-7. doi:10.1155/2015/945262

[2] Sibley C, Yazici Y, Tascilar K, Khan N, Bata Y, Yazici H, et al. Behçet syndrome manifestations and activity in the United States versus Turkey - a cross-sectional cohort comparison. J Rheumatol 2014;41:137984 doi:10.3899/jrheum.131227

[3] Bonitsis NG, Nguyen LBL, LaValley MP, Papoutsis N, Altenburg A, et al. Gender-specific differences in Adamantiades-Behçet's disease manifestations: an analysis of the German registry and meta-analysis of data from the literature. Rheumatology (Oxford). 2015;54: 121-33. doi:10.1093/rheu matology/keu247

Disclosure of Interest: None declared

DOI: 10.1136/annrheumdis-2018-eular.3249

\section{THU0629 A STUDY OF WBMRI IN ESTIMATING DISEASE ACTIVITY OF PM/DM AND THE FOLLOWING DISEASES DIAGNOSIS}

Y. Li, D. Jiang, X. Zheng, L. Lu, Z. Li, Y. Guo, J. Tang, X. He, D. Xu, G. Gao. the First Affiliated Hospital of Zhengzhou University, Zhengzhou, China

Background: IM is a group of disease characterised by chronic symmetrical myasthenia, muscle fatigue and monocyte infiltrating skeletal muscle. This disease usually associates with the muscle of trunk and proximal limbs, skin lesions companied or not. Dermatomyositis (DM) and polymyositis (PM) account for the major part of IM clinically. The diagnose of these diseases mainly rely on clinical manifestation, biopsy, ECG and serum creatase. Whole-body magnetic resonance imaging (WBMRI) has been reported succeeding in diagnosing PM/DM through short tau inversion recovery (STIR) sequence, but the ability to estimate disease activity hasn't been reported.

Objectives: To evaluate the value in WBMRI through STIR sequence in evaluating the disease activity of polymyositis (PM) and dermatomyositis (DM) and screening interstitial lung disease (ILD) and osteonecrosis. 